\title{
Faster magnetic resonance imaging in emergency room patients with right lower quadrant pain and suspected acute appendicitis
}

\author{
Memoona Mian $1,2 A, B, C, D, E, F$, Faisal Khosa ${ }^{1 A, E, F}$, Ismail T. Ali ${ }^{1,3 A, B, C, D}$, Patrick McLaughlin ${ }^{1 A, B, E}$, Darren Klass ${ }^{1 A}$, \\ Silvia Chang ${ }^{1 \mathrm{~A}}$, Savvas Nicolaou ${ }^{1 \mathrm{~A}, \mathrm{~B}, \mathrm{C}, \mathrm{F}}$ \\ 'Department of Radiology, Vancouver General Hospital, Vancouver, Canada \\ 2Department of Radiology, University of Arkansas for Medical Sciences, Little Rock, USA \\ ${ }^{3}$ Sunny Brook Health Sciences Centre, Toronto, Canada
}

\section{Abstract}

Purpose: Emergency Departments (ED) are becoming busier, with a resultant increase in the number of imaging referrals. The purpose of this study was to assess the diagnostic accuracy of an abbreviated two sequence magnetic resonance (MR) protocol for evaluating ED patients with right lower quadrant pain and suspected acute appendicitis, with a view to expediting patient turnaround times and imaging costs.

\begin{abstract}
Material and methods: Fifty patients (49 females, one male; mean age $25.4 \pm 5.2$ years) who underwent ED MR imaging from July 2014 to March 2015 for right lower quadrant pain were retrospectively reviewed. MR abdomen/pelvis was performed on 1.5 T MR obtaining axial T1 gradient echo in/out of phase, transverse fast spin echo T2 with fat sat/ motion correction, axial/coronal T2 HASTE (half-Fourier acquisition single-shot turbo spin-echo), and axial DWI (diffusion-weighted imaging) sequences. Images were reviewed by two fellowship-trained radiologists on a five-point confidence scale. Mean acquisition/interpretation times for the standard departmental protocol and the proposed abbreviated MR protocol (comprising T2 HASTE and DWI images) were calculated. Sensitivity, specificity, and diagnostic accuracy for the abbreviated protocol against the full protocol were also calculated.
\end{abstract}

Results: Mean scanning time for abbreviated protocol and standard protocol was calculated to be 21.1 minutes and 40.5 minutes, respectively. Mean interpretation time for abbreviated protocol for reader one and two was $4.1 \pm 1.5$ minutes and $4.5 \pm 1.4$ minutes, respectively, and for standard protocol was $8.1 \pm 1.8$ minutes and $7.1 \pm 1.4$ minutes, respectively. Sensitivity, specificity, and accuracy for the FAST protocol were calculated to be $100 \%$ each for reader one and $75 \%, 100 \%$, and $94 \%$, respectively, for reader two.

Conclusions: The proposed abbreviated MR protocol has comparable diagnostic accuracy in diagnosing ED patients with right lower quadrant pain, with significant reduction in imaging/interpretation times. It thus has the potential to be implemented in ED imaging with significant reduction in patient turnaround times and costs.

Key words: magnetic resonance imaging, acute appendicitis, right lower quadrant pain, diffusion weighted imaging, emergency.

\section{Introduction}

Emergency rooms are becoming busier day by day. Resultantly, the numbers of emergency department (ED) imag- ing studies that need to be done are also on the rise. Patient turnaround time is an essential component of workflow in the $\mathrm{ED}$, and it continues to gain greater importance due to worsening ED crowding and increasing waiting times [1]. Referring physicians often believe that emergency depart-

Correspondence address:

Memoona Mian, MD, FRCR, Department of Radiology, University of Arkansas for Medical Sciences, 4301 West Markham St., Little Rock, AR 72205, USA,

phone: +1-501-478-5559, fax: 501-603-1596, e-mail: drmmian@hotmail.com

Authors' contribution:

A Study design · B Data collection · C Statistical analysis · D Data interpretation · E Manuscript preparation · F Literature search · G Funds collection 
ment crowding is partially caused by delayed or redundant radiology services [2]. Various studies done previously have evaluated utilisation of different imaging services in the ED and have raised potential concerns about possible inappropriate use of imaging in the $\mathrm{ED}$, associated costs, radiation dose, and potential contribution to crowding in the ED [3].

Right lower quadrant pain remains a common ED presentation with the most important differential diagnosis being acute appendicitis. Additional pathologies such as pyelonephritis and ovarian abnormalities etc. can also be encountered. Prompt diagnosis/exclusion have significant management implications in the urgent care setting. With almost universal availability, inexpensiveness, and lack of ionising radiation, ultrasound is usually considered the initial imaging modality. However, the sensitivity and specificity can vary due to the patient body habitus, expertise of the sonographer, and even the position of the cecum. This becomes even more challenging in gravid females [4]. Computed tomography (CT) has a significantly higher diagnostic yield for appendicitis when compared with ultrasound $[5,6]$. However, concerns about ionising radiation exposure make it less desirable in young and/or pregnant females.

Relatively high cost of magnetic resonance imaging (MRI), its limited availability, and long imaging times have been impediments to its utilisation in the ED. However, in recent times, technological developments have made ultrafast sequences possible, resulting in shorter exam times and fewer patient motion artefacts [7]. Also, lack of ionizing radiation makes it a popular and safe imaging method in young/pregnant females. The utility of MRI for diagnosing acute appendicitis has been extensively studied in the past [8]. Diffusion-weighted imaging (DWI) has also been evaluated as an adjunct to basic MRI sequences for the diagnosis of acute appendicitis, with proven benefit. Multiple studies have been performed to assess and verify the improved diagnostic accuracy of DWI for acute appendicitis, with surgical diagnosis as the gold standard [9-11]. However, obtaining a complete MRI abdomen/pelvis with additional DWI can result in long scan times.

The aim of our study was to assess the diagnostic performance of a proposed abbreviated two-sequence MR protocol comprising DWI and T2 HASTE (half-Fourier acquisition single-shot turbo spin-echo), which could potentially be performed exclusively in ED patients presenting with right lower quadrant pain, with a view to improving radiology turnaround times and reducing costs while maintaining diagnostic accuracy.

\section{Material and methods}

\section{Hypothesis}

Radiological interpretation of an abbreviated two-sequence MR protocol comprising DWI and T2 HASTE images re- sults in a significant decrease in imaging and interpretation times without compromising diagnostic accuracy when compared with a full MR protocol in ED patients presenting with right lower quadrant pain and suspected acute appendicitis.

\section{Patient population}

Institutional review board ethics approval and a waiver of informed consent were obtained for this retrospective study. Our institution's Clinical Data Warehouse was interrogated to identify all consecutive patients who presented to the emergency room at Vancouver General Hospital (Vancouver, British Columbia, Canada) between July 2014 and March 2015 with right lower quadrant pain and had an MRI abdomen/pelvis done for a clinical diagnosis of possible acute appendicitis. Inclusion criteria were as follows: adult female and male patients (more than 18 years of age) presenting to the ED with right lower quadrant pain and primary concern for acute appendicitis; gravid females with right lower quadrant pain but no vaginal bleeding. Paediatric patients and gravid females with right lower abdominal pain and associated vaginal bleeding were excluded for the purpose of this study. Our study included a total of 50 patients of reproductive age (49 females, one male).

Initial ED ultrasound exam was performed for some patients; however, the ultrasound results were not reviewed in detail for the purpose of this study because the aim was primarily to assess the diagnostic performance of a shorter MR protocol versus the standard full departmental protocol, taking the full protocol results as a gold standard for the purpose of the study.

\section{Image acquisition}

Abdominal/pelvic MRI exams were performed in supine position with an Aera 1.5 Tesla MRI (Siemens AG Medical Solutions, Erlangen, Germany) using a body coil. No intravenous/oral contrast or bowel relaxant was given for the scans. The maximum gradient force of the superconducting magnet was $30 \mathrm{mT} / \mathrm{m}$, and the maximum field of view (FOV) width was $400 \mathrm{~mm}$. All patients underwent the following imaging protocol.

Axial gradient echo in-out of phase T1-weighted images (TR, $11.5 \mathrm{~s}$; TE, $4.77 \mathrm{~s}$; flip angle, $10^{\circ}$; matrix, $320 \times 320$; slice thickness, $3 \mathrm{~mm}$; slice gap, 20\%), transverse FSE T2-weighted images with fat sat/motion correction (TR between 4583 and $6836 \mathrm{~s}$; TE, $86 \mathrm{~s}$; flip angle, $155^{\circ}$; matrix, $320 \times 320$; slice thickness, $3 \mathrm{~mm}$; slice gap, $20 \%$ ), axial T2 HASTE images (TR, $1400 \mathrm{~s}$, TE, $90 \mathrm{~s}$; slice thickness, $3 \mathrm{~mm}$; slice gap, 20\%), and coronal T2 HASTE images (TR, $1400 \mathrm{~s}$; TE, $127 \mathrm{~s}$; slice thickness, $3 \mathrm{~mm}$; slice gap, 20\%) with breath holding technique. Axial DWI/ADC maps were also obtained for all scans. The diffusion weighted single-shot echo-planar sequence and chemical shift fat suppression 
were obtained without contrast media and without breath hold (TR/TE, 7600/91; matrix, $192 \times 144$; slice thickness, $5 \mathrm{~mm}$; slice gap, 20\%; FOV, $320 \mathrm{~mm}$; PAT, factor, GRAPPA). The protocol used for echo-planar DWI at our institution was as follows: $0 \mathrm{~s} / \mathrm{mm}^{2}, 50 \mathrm{~s} / \mathrm{mm}^{2}, 400 \mathrm{~s} / \mathrm{mm}^{2}, 1000 \mathrm{~s} / \mathrm{mm}^{2}$, and $\mathrm{ADC}$.

\section{Image analysis}

In a randomised blinded fashion, two board-certified and fellowship-trained radiologists with $>5$ years' experience in emergency radiology independently reviewed images for each patient for the presence/absence of acute appendicitis with regards to interpretation confidence on a five-point scale (Table 1). Only the T2 HASTE and DWI images were reviewed in the first setting to establish a diagnosis based on the abovementioned five-point scale. This was subsequently followed by reviewing the entire set of images including the T1W, FSE T2W images, DWI, and T2 HASTE images. The time gap between the two sets of readings was 60 days to avoid bias. The criteria we used for diagnosing acute appendicitis included: appendix
Table 1. Point interpretation confidence scale for detecting the appendix and making diagnosis

\begin{tabular}{|l|c|}
\hline Appendix not confidently visualised/Non-diagnostic study & 1 \\
\hline Low confidence in diagnosis & 2 \\
\hline Low-intermediate confidence & 3 \\
\hline Intermediate confidence & 4 \\
\hline High confidence & 5 \\
\hline
\end{tabular}

diameter $>9 \mathrm{~mm}$, appendicular wall thickness $>2 \mathrm{~mm}$, hyperintense appearances on DWI (Figures 1 and 2), caecal pole thickening, and surrounding oedema/fluid or abscess formation [8,9]. Any additional positive findings that could account for the patient's symptoms were also documented for both subsets.

\section{Statistical analysis}

The defining statistics related to the characteristics of interest were expressed as mean, median, standard devia-
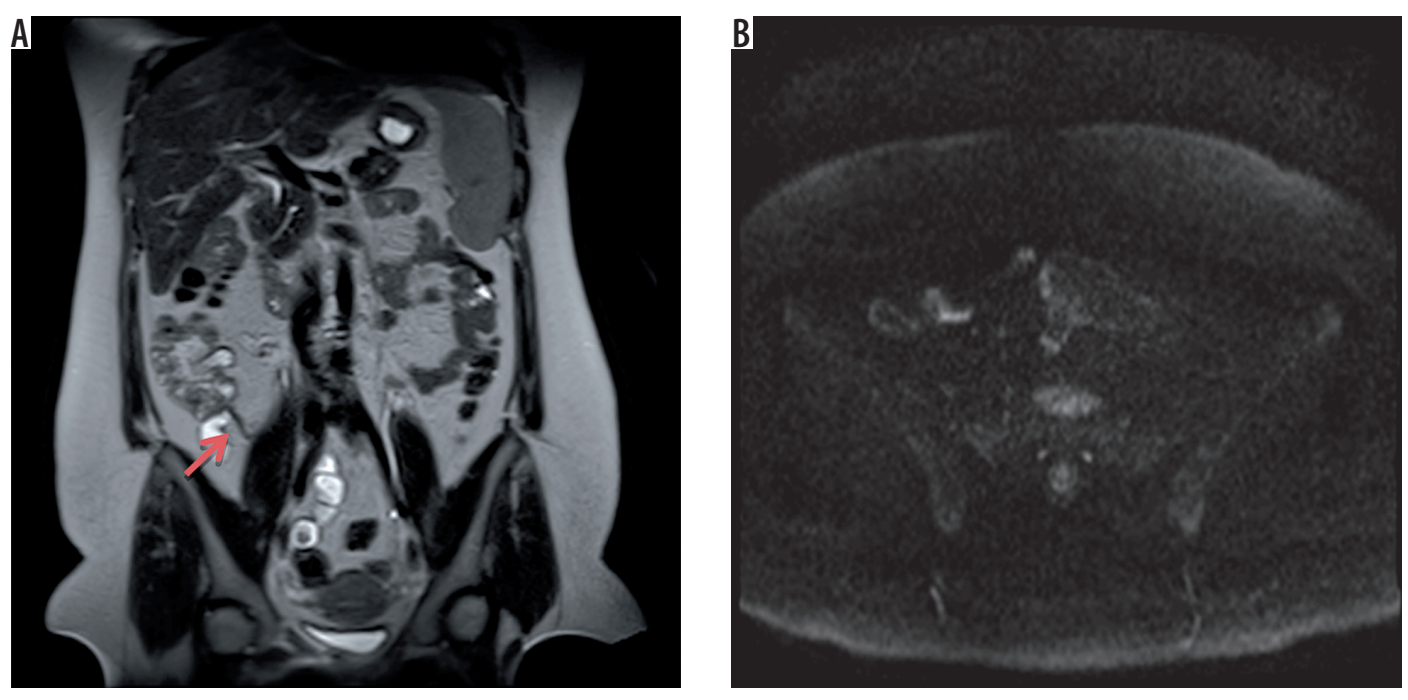

Figure 1. 25-year-old female with right lower quadrant pain and elevated white blood cell. A) Coronal T2W image shows normal appearance of the appendix with normal periappendiceal fat (arrow). B, C) Axial DW/ADC images do not show restricted diffusion in the appendix/periappendeceal fat, excluding appendicitis

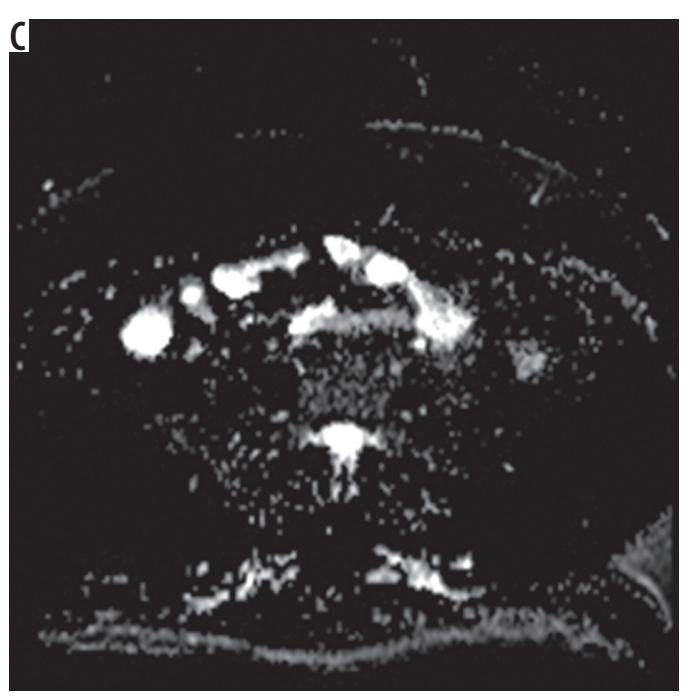



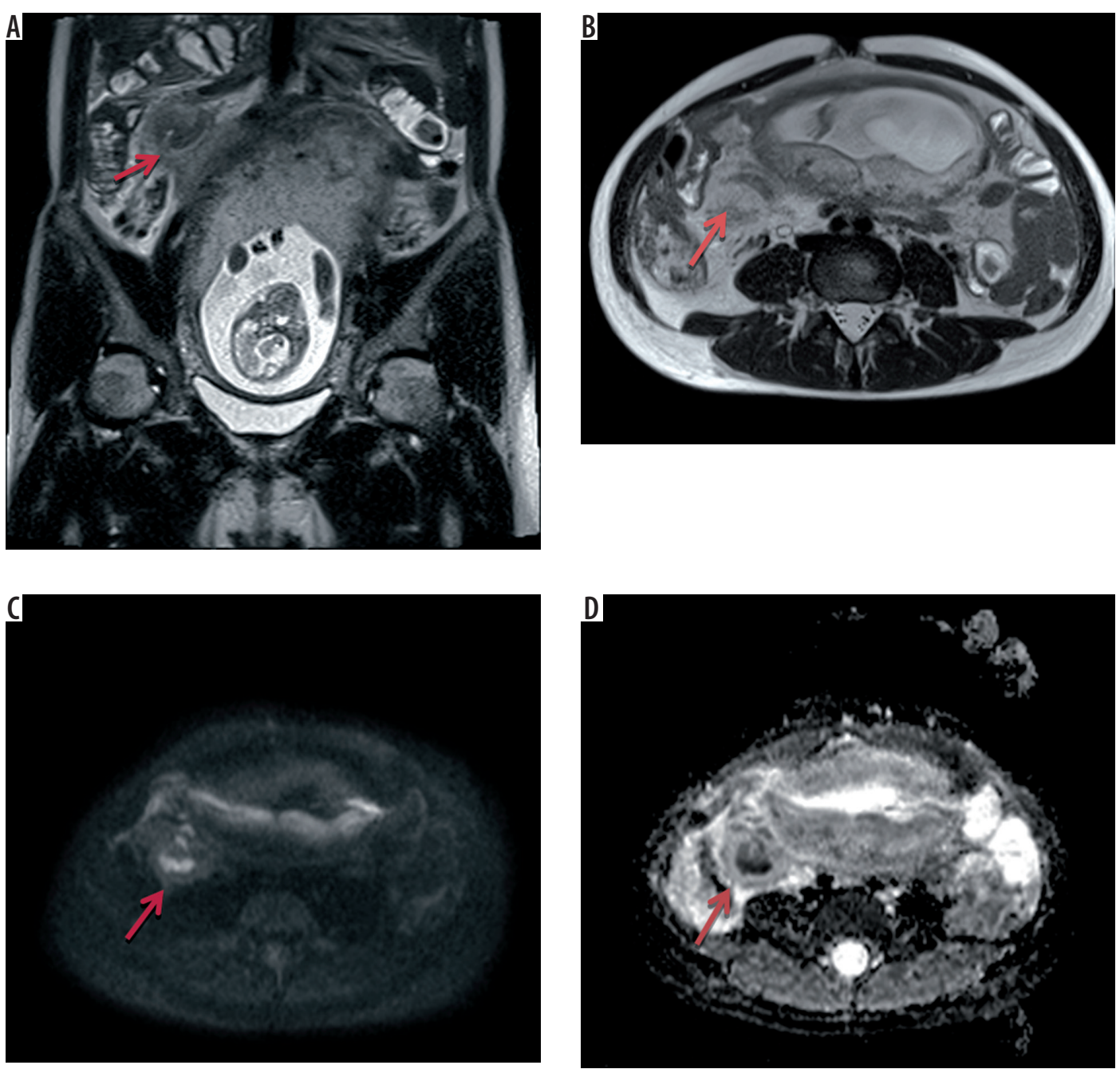

Figure 2. 35-year-old 20 weeks pregnant female with repeated episodes of right lower quadrant pain. Appendicitis? A, B) Coronal and axial T2W images show dilated appendix with periappendeceal soft tissue mass and fat stranding adjacent to the gravid uterus (arrow) which also shows restricted diffusion (arrow) on the DW/ADC images (C, D). Findings are consistent with subacute appendicitis

tion, and minimum and maximum values. Inter-observer variability between the interpretations of the two blinded readers was calculated and kappa values were determined. A $p$ value of $<0.05$ was considered statistically significant. Calculations were performed using commercially available software (Statistical Package for Social Sciences, Version 21.0, SPSS Inc., Chicago, Illinois, USA). The mean acquisition and interpretation times for the entire set of images including T1W, FSE T2W images, DWI, and T2 HASTE images were calculated. Subsequently, the scan acquisition and interpretation times for only the T2 HASTE and DWI were calculated as the second proposed faster ED imaging protocol in our patient subset. Sensitivity, specificity, and diagnostic accuracy for the abbreviated subset of images was calculated against the complete MR protocol diagnoses.

\section{Results}

The mean age of the study population was $25.4 \pm 5.2$ years. Fourteen patients were pregnant at the time of imaging.

\section{Imaging results}

Mean scan/table time for the complete image set acquisition was calculated to be 40.5 minutes whereas the mean scan/table time for the T2 HASTE and DWI subset was 21.1 minutes. Mean interpretation time for the complete protocol was $8.1 \pm 1.8$ minutes for reader one and 7.1 \pm 1.4 minutes for reader two. The mean interpretation time for the abbreviated subset was $4.1 \pm 1.5$ minutes for reader one and $4.5 \pm 1.4$ minutes for reader two. The mean times for both these subsets were found to be significantly different $(p<0.05)$.

The appendix was not identified on three scans which were considered negative for the purpose of our study given the absence of indirect signs of inflammation in the right lower quadrant (no fat stranding or free fluid, DWI negative, and lack of caecal pole thickening). None of these three cases was operated upon, and none had a documented complication upon clinical follow-up (chart review at 30-day patient follow-up with the primary team was done to see how the patients were subsequently), con- 

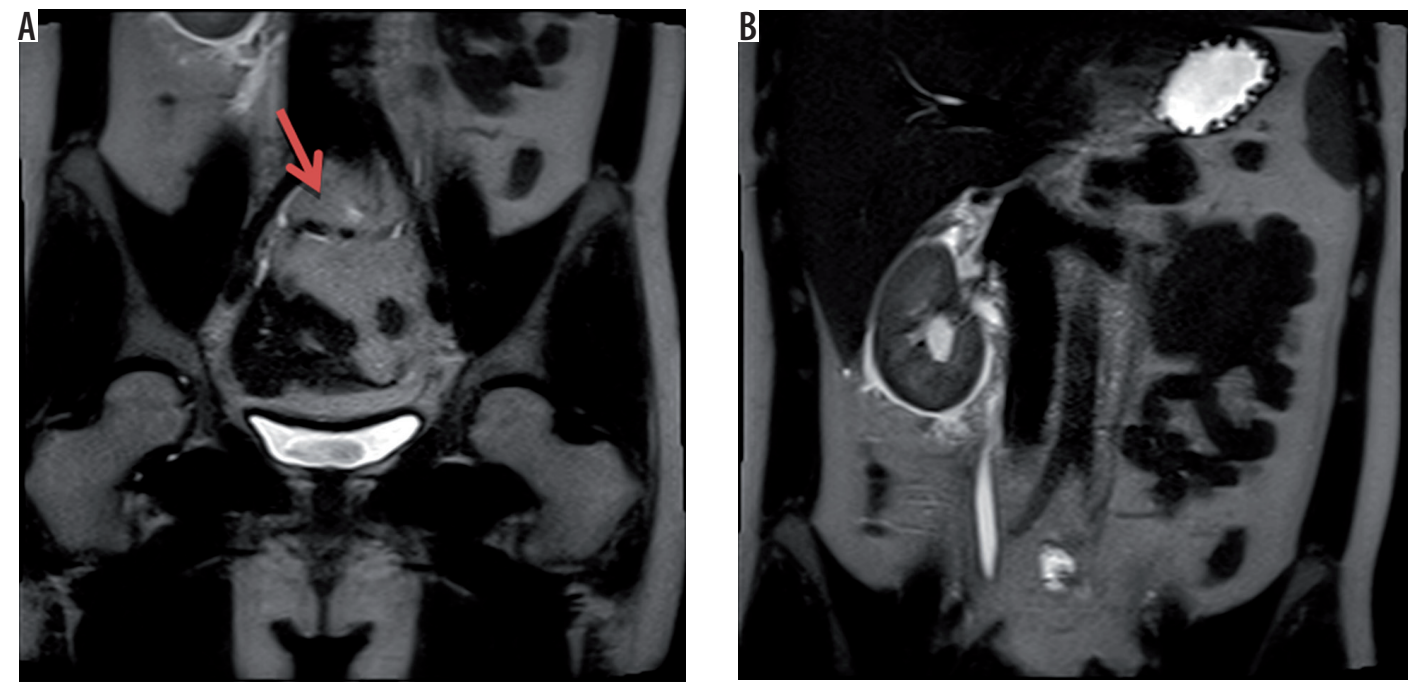

Figure 3. 28 year-old-female with right lower abdominal pain and leukocytosis. A) T2W coronal image shows normal appearance of the appendix (arrow). However, there is significant right perirenal fluid and hydroureter $(B)$ consistent with acute pyelonephritis, accounting for the patients symptoms

sistent with our negative diagnosis. Interobserver reliability was found to be kappa $0.259(p<0.005)$, indicating a significant but fair degree of agreement in diagnosing or excluding appendicitis on the complete image set. The interobserver reliability for the abbreviated subset comprising of T2HASTE and DWI was found to be kappa 0.320 $(p<0.005)$, indicating significant but fair agreement between the two observers in diagnosing/excluding appendicitis. Three patients were identified to have pyelonephritis (Figure 3) and two had complex adnexal cysts as possible causes for their clinical symptoms. These were identified by both readers on both image groups.

Initial ultrasound exams were done in 23 patients. The detailed results of these ED ultrasound exams or the final histopathology results for the operated cases were not reviewed for the purpose of this study because the primary aim was to compare the diagnostic performance of the two MR protocol subsets, considering the full protocol interpretation as a gold standard for the purpose of this study.

\section{Statistical evaluation}

Sensitivity and specificity for the abbreviated MR protocol comprising of T2 HASTE and DWI was calculated to be
$100 \%$ for reader one and $75 \%$ for reader two. The diagnostic accuracy for the abbreviated protocol was $100 \%$ and $94 \%$ for both observers. The Wilcox rank sum test was used to analyse the level of confidence of the radiologists for both imaging sets. No statistically significant difference $(p=0.623)$ in the level of confidence of the observers in the interpretation of the complete and abbreviated protocol was found (Table 2). However, the mean interpretation time difference was found to be significant.

\section{Discussion}

The versatility of the technique, lack of intravenous contrast, and the increasing awareness of radiation-related health risks associated with $\mathrm{CT}$ have resulted in more frequent use of MRI in the diagnostic workup for several acute abdominal conditions, including acute appendicitis [12]. There have been recent reports bringing concern that intravenous iodinated contrast in combination with ionising radiation might increase damage to the DNA double helical strands, thus increasing the likelihood of DNA mutation and malignancies [3]. This lends more evidence that we should try to utilise other modalities when possible, particularly in paediatric, pregnant, and young

Table 2. Five-point confidence scale interpretation results for standard and fast protocols for the two readers

\begin{tabular}{|l|c|c|c|c|}
\hline \multirow{2}{*}{ Confidence Scale } & \multicolumn{2}{|c|}{ Standard protocol } & \multicolumn{2}{c|}{ Fast protocol } \\
\cline { 2 - 5 } & Reader 1 & Reader 2 & Reader 1 & Reader 2 \\
\hline Not visualized & 2 & 3 & 4 & 3 \\
\hline Low confidence & 1 & 1 & 1 & 1 \\
\hline Low-intermediate confidence & 4 & 4 & 3 & 5 \\
\hline Intermediate confidence & 4 & 5 & 6 & 37 \\
\hline High confidence & 39 & 37 & 36 & 5 \\
\hline
\end{tabular}


adult populations. As MRI becomes increasingly available in emergency settings [13], its use for evaluating all patients with acute right lower quadrant pain is expected to increase.

According to the American College of Radiology (ACR) Appropriateness Criteria, patients with right lower quadrant pain and suspected acute appendicitis should ideally be imaged with CT scan of the abdomen and pelvis; however, MR imaging may be considered more appropriate in cases with iodinated contrast allergy or poor renal function. In pregnant patients presenting with similar symptoms, ACR appropriateness guidelines favour non-contrast enhanced MR imaging over CT scanning due to radiation hazards to the foetus. The ACR Appropriateness Guidelines highlight the need for further research regarding MR utility in the general population for evaluation of acute appendicitis because limited evidence is available to date. They also mention longer imaging times as a potential shortcoming of MR imaging for ED utility.

The utility of MR DWI has been extensively studied in the recent past, with innumerable studies highlighting its utility in the diagnosis of many disease conditions, including hepatic, urinary, and pelvic malignancies [14-16]. Literature supports its utility in the diagnosis of acute appendicitis as well, with multiple published studies from various institutions across the world validating its advantage in such cases [10]. The inflammatory process of acute appendicitis alters the structural organisation of the appendix and the adjacent tissue, thereby affecting the diffusivity of water molecules, which results in increased signal intensity on DWI [4]. The primary purpose of our study was not simply to evaluate the accuracy of DWI in diagnosing potential acute appendicitis (this has already been proven in the past to be beneficial) but also to see if we could propose a modified abbreviated MR protocol incorporating DWI, exclusively for ED patients with right lower quadrant pain with the idea of saving time, reducing imaging costs, and improving ED turnaround times without compromising the radiologist's diagnostic confidence. We wanted to evaluate if there was any significant decrease in the confidence of the reading radiologists or the imaging diagnosis generated from the abbreviated protocol when compared with interpretation of a full five-sequence set of MR images currently obtained in such patients in our department. Our current full MR protocol to the best of our knowledge is comparable to the standard MR protocol adopted in most radiology departments. A study done by Inci et al. quantitatively evaluated DW signal intensities and ADC values for normal and inflamed appendixes and reported a sensitivity of $99 \%$ and a specificity of $97 \%$ with DWI using a cut-off value of 56 for the signal intensity. These findings correlated with the histopathological results and, except for one patient with histologically proven appendicitis in their study group comprising 119 patients, all inflamed appendixes were hyperintense on DWI (98.7\%) [10]. We also used
DWI hyperintensity as a criterion for suggesting acute appendicitis in our study group. Avcu et al. also studied the effectiveness of DWI and ADC values in differentiating perforated and non-perforated appendicitis in a group of 60 patients, where $40 / 44$ patients with a radiological diagnosis of acute appendicitis underwent surgery, confirming 12 cases of perforated and 28 cases of non-perforated acute appendicitis. The sensitivity, specificity, and diagnostic accuracy rate of DWI in their study was $97.5 \%$, $100 \%$, and $98.1 \%$, respectively [9]. The diagnostic accuracy of our abbreviated protocol incorporating DWI was found to be comparable, being $94 \%$ and $100 \%$ for the two readers.

A study conducted by Leewenburgh et al. compared radiologist interpretation sensitivity with conventional MR images without and with the addition of DWI sequences and demonstrated an improvement in sensitivity from 0.80 to $0.87(p<0.001)$ in all readings combined when DW images were read in addition to the conventional MR images [17]. However, the addition of DWI to current MRI protocols to improve sensitivity results in additional imaging and increased reporting times. A combination of DWI and conventional MRI was found to be the most sensitive and most accurate technique for the diagnosis of acute appendicitis in children in another study conducted by Bayraktutan et al. [11]. They found that the combination had a sensitivity and accuracy of 0.92 and 0.92 , respectively, versus 0.78 and 0.77 with DWI alone and 0.81 and 0.82 with conventional MRI alone. We thus tried to evaluate an abbreviated protocol that incorporated both DWI and T2W images.

In addition to evaluation for appendicitis, MRI facilitates identification of potential alternate diagnoses to explain the patient's symptoms, as well. Because MRI for right lower quadrant pain usually includes imaging through most (or all) of the abdomen and pelvis, it allows comprehensive abdominopelvic imaging evaluation rather than focused evaluation as in ultrasound, and that too without the radiation hazard as in CT. Also, the inherent soft-tissue contrast resolution of MRI allows greater detection of ovarian pathology than CT, including ruptured haemorrhagic cysts [18]. Studies from three institutions report the rate of alternate diagnosis detection at $20.6 \%$ [19], 19.2\% [20], and 18.4\% [18]. In all these studies, the two most frequent diagnoses were adnexal cysts and enteritis/colitis, each at approximately 6\%. MRI also demonstrated ovarian torsion, pyelonephritis, obstructive urolithiasis, and even malignancy, among others. In our study, the complete MR exam detected a possible alternate diagnosis in five patients who had a normal appendix in our study group. All five were also identified on the proposed abbreviated protocol.

MRI is now commonly recommended for appendicitis evaluation in the setting of pregnancy, and Fonseca et al.s [21] 2014 findings might be pertinent to the discussion here because $14 / 50$ of our study patients were pregnant at 
the time of ED presentation/imaging. Fonseca et al. [21] reported a retrospective review of the records of 79 pregnant patients who underwent sonography, and 31 of those also had MRI. All pathways resulted in high specificity; however, sensitivity was $25 \%$ for clinical diagnosis, $39 \%$ for US, and $100 \%$ for MRI [21]. Their results also demonstrated that the patients undergoing MRI were more frequently discharged from the emergency department (odds ratio $0.35 ; p=0.04)$ and had shorter length of stay $(33.7 \mathrm{~h}$ vs. $64.8 \mathrm{~h}, p<0.001)$ than patients without MRI. Mean hospital charges were less when MRI was used in patient groups both with and without appendicitis. Mean total hospital charges at their institution were $\$ 8174$ with appendicitis and MRI vs. $\$ 8544$ with appendicitis and no MRI; and \$3,205 with no appendicitis and MRI vs. \$3,615 with no appendicitis and no MRI. These authors concluded that MRI shortened the length of stay without increasing hospital charges in pregnant patients [21]. We believe that our proposed abbreviated MR imaging protocol including DWI and T2 HASTE images can also result in significant imaging cost reduction because the radiologist's interpretation was found to be similar to what it would have been with a full five-sequence MR exam.

Although the results are promising, they are still incomplete. The limitations of our study include a retrospective design, a small sample size, and a highly specific patient population. We did not incorporate final histopathology results for the operated cases; reviewing those could have potentially further validated the utility of the proposed abbreviated protocol in the ED setting for such patients. A detailed cost analysis incorporating imaging, hospital stay, etc. would be prudent to further support our hypothesis and convince other radiologists and physicians to adopt limited scanning in ED patients to facilitate faster triage and improve patient waiting and turnaround times. The proposed technique can be further studied using a prospective design and a larger patient population in a multicentre setting for further validation.

\section{Conclusions}

The proposed abbreviated two-sequence MR exam comprising T2W HASTE and DWI images has a high diagnostic accuracy in detecting / excluding appendicitis with no significant difference in the level of confidence of the reading radiologist when compared with the full multisequence MR protocol. It can be a valuable tool in the emergency department for assessing patients with right lower quadrant pain and possible acute appendicitis with significant reduction in imaging/ interpretation times as well as costs.

\section{Acknowledgments}

We would like to thank Dr. Nizar Noor Ali for his efforts and help with the statistical analysis and results section of this study. This research article would not have been possible without his efforts.

This work has been presented at the RSNA annual meeting 2015.

\section{Conflict of interest}

The authors report no conflict of interest.

\section{References}

1. De Florio R, Coughlin B, Coughlin R, et al. Process modification and emergency department radiology service. Emerg Radiol 2008; 15: 405-412.

2. Shuaib W, Vijayasarathi A, Tiwana MH, et al. The diagnostic utility of rib series in assessing rib fractures. Emerg Radiol 2014; 21: 159-164.

3. Piechowiak El, Peter JF, Kleb B, et al. Intravenous Iodinated Contrast Agents Amplify DNA Radiation Damage at CT. Radiology 2015; 275: 692-697.

4. Puylaert JB. Ultrasound of the acute abdomen: gastrointestinal conditions. Radiol Clin North Am 2003; 41: 1227-1242.

5. Binnebosel M, Otto J, Stumpf M, et al. Acute appendicitis. Modern diagnostics - surgical ultrasound. Chirurg 2009; 80: 579-587.

6. Doria AS, Moineddin R, Kellenberger CJ, et al. US or CT for diagnosis of appendicitis in children and adults? A meta-analysis. Radiology 2006; 241: 83-94.

7. Pedrosa I, Levine D, Eyvazzadeh AD, et al. MR imaging evaluation of acute appendicitis in pregnancy. Radiology 2006; 238: 891-899.

8. Barger R, Nandalur K. Diagnostic performance of magnetic resonance imaging in the detection of appendicitis in adults: a metaanalysis. Acad Radiol 2010; 17: 1211-1216.
9. Avcu S, Cetin FA, Arslan H, et al. The value of diffusion-weighted imaging and apparent diffusion quantification in the diagnosis of perforated and nonperforated appendicitis. Diagn Interv Radiol 2013; 19: 106-110.

10. Inci E, Kilickesmez O, Hocaoglu E, et al. Utility of diffusion-weighted imaging in the diagnosis of acute appendicitis. Eur Radiol 2011; 21: 768-775

11. Bayraktutan U, Oral A, Kantarci M, et al. Diagnostic performance of diffusion-weighted MR imaging in detecting acute appendicitis in children: comparison with conventional MRI and surgical findings. J Magn Reson Imaging 2014; 39: 1518-1524.

12. Birnbaum BA, Wilson SR. Appendicitis at the millennium. Radiology 2000; 215: 337-339.

13. Lally KP, Cox CS Jr, Andrassy RJ. Appendix. In: Sabiston textbook of surgery. Townsend CM Jr, Mattox KL, Evers BM, et al. (eds.). Sabiston textbook of surgery. $17^{\text {th }}$ ed. Saunders, New York 2004; 1381-1395.

14. Naganawa S, Sato C, Kumada H, et al. Apparent diffusion co-efficient in cervical cancer of the uterus: comparison with the normal uterine cervix. Eur Radiol 2005; 15: 71-78. 
15. Tamai K, Koyama T, Saga T, et al. Diffusion-weighted MR imaging of uterine endometrial cancer. J Magn Reson Imaging 2007; 26 : 682-687.

16. Kilickesmez O, Atilla S, Soylu A, et al. Diffusion-weighted imaging of the rectosigmoid colon: preliminary findings. J Comput Assist Tomogr 2009; 33: 863-866.

17. Incesu L, Coskun A, Selcuk MB, et al. Acute appendicitis: MR imaging and Sonographic Correlation. AJR Am J Roentgenol 1997; 168: 669-674.

18. Dillman J, Gadepalli S, MD, Sroufe N, et al. Equivocal pediatric appendicitis: unenhanced MR imaging protocol for nonsedated chil- dren - a clinical effectiveness study. Radiology 2015; doi:10.1148/ radiol.2015150941

19. Koning JL, Naheedy JH, Kruk PG. Diagnostic performance of contrast-enhanced MR for acute appendicitis and alternative causes of abdominal pain in children. Pediatr Radiol 2014; 44: 948-955.

20. Moore MM, Kulaylat AN, Brian JM, et al. Alternative diagnoses at paediatric appendicitis MRI. Clin Radiol 2015; 70: 881-889.

21. Fonseca AL, Schuster KM, Kaplan LJ, et al. The use of magnetic resonance imaging in the diagnosis of suspected appendicitis in pregnancy: shortened length of stay without increase in hospital charges. JAMA Surg 2014; 149: 687-693. 